\title{
Statistical Mechanics where Newton's Third Law is Broken
}

\author{
A. V. Ivlev, ${ }^{1, *}$ J. Bartnick, ${ }^{2}$ M. Heinen, ${ }^{2,3}$ C.-R. Du, ${ }^{4}$ V. Nosenko, ${ }^{5}$ and H. Löwen ${ }^{2}$ \\ ${ }^{1}$ Max-Planck-Institut für Extraterrestrische Physik, 85741 Garching, Germany \\ ${ }^{2}$ Institut für Theoretische Physik II, Weiche Materie, Heinrich-Heine-Universität, \\ 40225 Düsseldorf, Germany \\ ${ }^{3}$ Division of Chemistry and Chemical Engineering, California Institute of Technology, \\ Pasadena, California 91125, USA \\ ${ }^{4}$ College of Science, Donghua University, Shanghai 201620, \\ People's Republic of China \\ ${ }^{5}$ Forschungsgruppe Komplexe Plasmen, Deutsches Zentrum für Luft- und Raumfahrt, \\ 82234 Weßling, Germany
}

(Received 4 March 2014; revised manuscript received 17 October 2014; published 26 March 2015)

\begin{abstract}
There is a variety of situations in which Newton's third law is violated. Generally, the action-reaction symmetry can be broken for mesoscopic particles, when their effective interactions are mediated by a nonequilibrium environment. Here, we investigate different classes of nonreciprocal interactions relevant to real experimental situations and present their basic statistical mechanics analysis. We show that in mixtures of particles with such interactions, distinct species acquire distinct kinetic temperatures. In certain cases, the nonreciprocal systems are exactly characterized by a pseudo-Hamiltonian; i.e., being intrinsically nonequilibrium, they can nevertheless be described in terms of equilibrium statistical mechanics. Our results have profound implications, in particular, demonstrating the possibility to generate extreme temperature gradients on the particle scale. We verify the principal theoretical predictions in experimental tests performed with two-dimensional binary complex plasmas.
\end{abstract}

DOI: 10.1103/PhysRevX.5.011035

\section{INTRODUCTION}

One of the fundamental postulates in physics is Newton's third law actio = reactio, laying the foundations of classical mechanics. This law, which states that the pair interactions between particles are reciprocal, holds not only for the fundamental microscopic forces but also for equilibrium effective forces on classical particles, obtained by integrating out microscopic degrees of freedom [1-5]. However, the action-reaction symmetry for particles can be broken when their interaction is mediated by some nonequilibrium environment: Such symmetry breaking occurs, for instance, when the environment moves with respect to the particles, or when a system of particles is composed of different species and their interaction with the environment is out of equilibrium. (Of course, Newton's third law holds for the complete "particles-plus-environment" system.) Recently, there have been numerous studies of nonreciprocal interactions on the mesoscopic length scale. Examples include forces induced by nonequilibrium

\footnotetext{
*ivlev@mpe.mpg.de
}

Published by the American Physical Society under the terms of the Creative Commons Attribution 3.0 License. Further distribution of this work must maintain attribution to the author(s) and the published article's title, journal citation, and DOI.
Subject Areas: Plasma Physics, Soft Matter, Statistical Physics fluctuations [6,7], optical [8,9] and diffusiophoretic $[10,11]$ forces, effective interactions between colloidal particles under solvent or depletant flow [12-15], shadow [16-18] and wake-mediated [19-21] interactions between microparticles in a flowing plasma, etc. A very different case of nonreciprocal interaction is "social forces" [22,23] governing, e.g., pedestrian dynamics.

A natural violation of Newton's third law in nonequilibrium environments can be easily illustrated. One example for nonreciprocal interactions occurs in the context of catalytically driven colloids (microswimmers) [11]. Typically, a single colloidal particle that produces or consumes chemicals on its surface, being embedded in a solution with a gradient in the chemical concentration, is propelled along the gradient- this nonequilibrium transport phenomenon is termed as diffusiophoresis. Since each particle generates an inhomogeneous concentration profile in its vicinity, the action-reaction symmetry in a binary mixture of microswimmers (with different mobilities and surface activities) is broken. The magnitude of the nonreciprocity can be tuned by varying the relative disparity of the activities and/or mobilities. The other very different system where the action-reaction symmetry is broken is quasi-two-dimensional (2D) binary complex plasmas $[21,24]$. Such complex plasmas are binary mixtures of charged microparticles, levitating in a discharge over a flat 
horizontal electrode at slightly different heights. The horizontal interactions between the microparticles are nonreciprocal because they are mediated by the plasma wakes-the perturbations below each particle, generated in a plasma streaming toward the electrode. The magnitude of the nonreciprocity is controlled by varying the difference of the levitation heights for the two species.

Nonreciprocal forces are, in principle, non-Hamiltonian (i.e., they cannot be derived from a classical many-body Hamiltonian), so the standard Boltzmann description of classical equilibrium statistical mechanics breaks down. Hence, it is a priori unclear whether concepts like temperature and thermodynamic phases can be used to describe them. Apart from a few considerations in the context of the multiscale coarse graining [4,25], the classical statistical mechanics of systems with nonreciprocal interactionsdespite their fundamental importance-remains widely unexplored.

In this paper, we present the statistical foundations of systems with nonreciprocal interparticle interactions. To describe various classes of interactions relevant to real experimental situations, we consider a generic model where the action-reaction symmetry is broken for the pair interaction between two subensembles. The asymmetry is characterized by the nonreciprocity parameter $\Delta$, which is the ratio of the nonreciprocal to reciprocal forces. We show that for the "constant" nonreciprocity, when $\Delta$ is independent of the interparticle distance $r$, one can construct a (pseudo) Hamiltonian with renormalized masses and interactions. Hence, being intrinsically nonequilibrium, such systems can nevertheless be described in terms of equilibrium statistical mechanics and exhibit detailed balance with distinct temperatures for different subensembles. (The temperature ratio is determined by $\Delta$.) For a general case, when $\Delta$ is a function of $r$, the system is no longer conservative-it follows a universal asymptotic behavior with the temperatures growing with time as $\propto t^{2 / 3}$. The temperature ratio is then determined by an effective constant nonreciprocity that is uniquely defined for a given interaction. The temperatures reach a steady state when the damping due to the surrounding medium is taken into account, while their ratio remains practically unchanged. One of the remarkable implications of our results is the occurrence of extreme temperature gradients, generated in mixtures of particles at the ultimate scale of interparticle distance.

To verify the principal theoretical predictions, we have also performed experimental tests with quasi-2D binary complex plasmas. The interaction of particles of one sort with the wakes generated by particles of the other sort leads to a very general mechanism of the action-reaction symmetry breaking, occurring due to the presence of a flow [as explained in detail in the caption of Fig. 3(c)]. This asymmetry makes 2D complex plasmas perfectly suited for studying generic properties of many-body systems with nonreciprocal interactions.

\section{RESULTS}

\section{A. Constant nonreciprocity and pseudo-Hamiltonian}

In the Introduction, we mentioned several prominent examples of nonreciprocity, including the situations when different particles interact differently with the surrounding nonequilibrium environment $[11,18]$ or when the actionreaction symmetry is broken in the presence of a flow $[12,21]$ (while the particles themselves may be identical). In what follows, for the sake of convenience, we generally attribute particles to "different species" when their pair interaction is nonreciprocal.

To describe the variety of nonreciprocal forces, we employ the following generic model: We consider a binary mixture of species $A$ and $B$, where the spatial dependence of the pair-interaction force is proportional to the derivative of the function $\varphi(r)$. The interaction is reciprocal for the $A A$ and $B B$ pairs, whereas between the species $A$ and $B$, the action-reaction symmetry is broken. The measure of the asymmetry is the nonreciprocity parameter $\Delta(\geq 0)$.

It is noteworthy that the only assumption made about the general form of isotropic nonreciprocal interactions is that nonpairwise additive many-body forces between different species are negligible (which is always justified to describe, e.g., complex plasmas and dilute colloids [21]). Apart from that, no further assumption is imposed on the model. Thus, the parameter $\Delta$ completely characterizes any isotropic type of pairwise nonreciprocal forces.

First, we consider the case when $\Delta$ is independent of the interparticle distance ("constant") - this situation represents, e.g., binary colloidal dispersions with the dominating diffusiophoretic interactions $[10,11]$ or complex plasmas with the shadow interactions [16-18]. We present the force $\mathbf{F}_{i j}$ exerted by the particle $i$ on the particle $j$ as follows:

$$
\mathbf{F}_{i j}=-\frac{\partial \varphi\left(r_{i j}\right)}{\partial \mathbf{r}_{j}} \times \begin{cases}1-\Delta & \text { for } i j \in A B \\ 1+\Delta & \text { for } i j \in B A \\ 1 & \text { for } i j \in A A \text { or } B B\end{cases}
$$

where $r_{i j}=\left|\mathbf{r}_{i}-\mathbf{r}_{j}\right|$ and each particle can be of the sort $A$ or $B$. In order to distinguish the effect of nonreciprocity, $\varphi(r)$ must be the same for the $A B$ and $B A$ pairs, while for the $A A$ and $B B$ pairs it may be different.

By writing the Newtonian equations of motion of individual particles interacting via the force (1), we notice that the interaction symmetry is restored if the particle masses and interactions are renormalized as follows:

$$
\tilde{m}_{i}=m_{i} \times \begin{cases}(1+\Delta)^{-1} & \text { for } i \in A \\ (1-\Delta)^{-1} & \text { for } i \in B\end{cases}
$$




$$
\tilde{\varphi}\left(r_{i j}\right)=\varphi\left(r_{i j}\right) \times \begin{cases}(1+\Delta)^{-1} & \text { for } i j \in A A \\ (1-\Delta)^{-1} & \text { for } i j \in B B \\ 1 & \text { for } i j \in A B \text { or } B A .\end{cases}
$$

Hence, a binary system (of $N$ particles) with nonreciprocal interactions of the form of Eq. (1) is described by a pseudoHamiltonian with the masses (2) and interactions (3). In particular, this fact implies the pseudomomentum and pseudoenergy conservation

$$
\begin{aligned}
\sum_{i}^{N} \tilde{m}_{i} \mathbf{v}_{i} & =\text { const } \\
\sum_{i}^{N} \frac{1}{2} \tilde{m}_{i} v_{i}^{2}+\sum_{i<j}^{N} \tilde{\varphi}\left(r_{i j}\right) & =\text { const }
\end{aligned}
$$

and allows us to employ the methods of equilibrium statistical mechanics to describe such systems. For instance, from equipartition $\frac{1}{2} \tilde{m}_{A}\left\langle v_{A}^{2}\right\rangle=\frac{1}{2} \tilde{m}_{B}\left\langle v_{B}^{2}\right\rangle \equiv \frac{1}{2} D k_{B} \tilde{T}$ (where $\tilde{T}$ is the pseudotemperature and $D$ is the dimensionality), it immediately follows that $T_{A}=(1+\Delta) \tilde{T}$ and $T_{B}=(1-\Delta) \tilde{T}$, i.e.,

$$
\frac{T_{A}}{T_{B}}=\frac{1+\Delta}{1-\Delta} .
$$

We conclude that mixtures of particles with nonreciprocal interactions, being intrinsically nonequilibrium, can nevertheless reach a remarkable state of detailed dynamic equilibrium, where the species have different temperatures $T_{A}$ and $T_{B}$. Note that the equilibrium is only possible for $\Delta<1$; otherwise, the forces $\mathbf{F}_{A B}$ and $\mathbf{F}_{B A}$ are pointed in the same direction [see Eq. (1)] and the system cannot be stable.

\section{B. General case and asymptotic universality}

Now, we shall study a general case, when the interactions between the species $A$ and $B$ are determined by the forces $\mathbf{F}_{A B, B A}(r)=\mp \mathbf{F}_{r}(r)+\mathbf{F}_{n}(r)$. The reciprocal $\mathbf{F}_{r}(r)$ and nonreciprocal $\mathbf{F}_{n}(r)$ components are arbitrary functions of the interparticle distance; they can always be presented as $\mathbf{F}_{r, n}(r)=(\mathbf{r} / r) F_{r, n}(r)$, where $F_{r, n}=-d \varphi_{r, n} / d r$. As we show below, the dynamic equilibrium is no longer possible in this case, and analytical results can only be obtained in certain limiting regimes.

To facilitate the analysis, we shall distinguish between the weakly and strongly coupled systems: The former regime represents the situation when binary collisions between particles play the dominant role ("dilute" systems), while in the latter regime, simultaneous interactions with many neighbors are crucial ("dense" systems). The transition between the regimes is determined by the coupling parameter $\Gamma$, which is the ratio of the mean energy of the (reciprocal) pair interaction to the particle thermal energy (i.e., $\Gamma \propto T^{-1}$ ).

Let us first study weakly coupled $(\Gamma \ll 1)$ systems whose dynamics is governed by binary interparticle collisions. (Numerical analysis of strongly correlated systems is presented in Sec. II D.) It is instructive to write the equations of motion for a pair of particles $A$ and $B$ in terms of the relative coordinate $\mathbf{r}=\mathbf{r}_{A}-\mathbf{r}_{B}$ and the center-of-mass coordinate $\mathbf{R}=\left(m_{A} \mathbf{r}_{A}+m_{B} \mathbf{r}_{B}\right) / M$,

$$
\begin{aligned}
M \ddot{\mathbf{R}} & =2 \mathbf{F}_{n}(r), \\
\mu \ddot{\mathbf{r}} & =\mathbf{F}_{r}(r)+\frac{m_{B}-m_{A}}{m_{A}+m_{B}} \mathbf{F}_{n}(r),
\end{aligned}
$$

where $\mu=m_{A} m_{B} /\left(m_{A}+m_{B}\right)$ and $M=m_{A}+m_{B}$ are the reduced and total masses, respectively. Using Eqs. (5) and (6), we calculate the variation of the kinetic energy after a collision $\delta E_{A, B}$, which is expressed via the relative velocity $\mathbf{v}=\dot{\mathbf{r}}$, the center-of-mass velocity $\mathbf{V}=\dot{\mathbf{R}}$, and the scattering angle $\chi$ for the relative motion (see Appendix A). In the approximation of small-angle scattering [26] $\chi \ll 1$, which significantly simplifies the analysis and is valid for sufficiently high kinetic energies (provided the pair interaction is not of the hard-sphere-like type), one can derive general equations describing the asymptotic evolution of the kinetic temperatures $T_{A, B}$. To obtain a closed-form solution, we assume that the elastic energy exchange in collisions provides efficient Maxwellization of the distribution functions (which can be verified by molecular-dynamics simulations discussed below). We derive the following equations for $2 \mathrm{D}$ systems:

$$
\begin{aligned}
\dot{T}_{A, B}= & \pm \frac{1 \pm \Delta_{\mathrm{eff}}}{1+\epsilon} \frac{\sqrt{2 \pi} n_{B, A} I_{r r}}{m_{A} m_{B}\left(\frac{T_{A}}{m_{A}}+\frac{T_{B}}{m_{B}}\right)^{3 / 2}} \\
& \times\left[\left(1+\Delta_{\mathrm{eff}}\right) T_{B}-\left(1-\Delta_{\mathrm{eff}}\right) T_{A}\right. \\
& \left.+\frac{\epsilon}{1 \pm \Delta_{\mathrm{eff}}}\left(T_{B}-T_{A}\right)\right]
\end{aligned}
$$

where $n_{\alpha}$ is the areal number density; for simplicity, below we assume $n_{A}=n_{B}=n$. The equations depend on the effective nonreciprocity $\Delta_{\text {eff }}$ and the interaction disparity $\epsilon$

$$
\Delta_{\mathrm{eff}}=I_{n n} / I_{r n}, \quad \epsilon=I_{r r} I_{n n} / I_{r n}^{2}-1 .
$$

The integrals $I_{\alpha \beta}=\int_{0}^{\infty} d \rho f_{\alpha} f_{\beta}$ are expressed via the scattering functions $f_{\alpha}(\rho)$ [see Eq. (A1) in Appendix A]; it is assumed that the integrals converge. We point out that $\Delta_{\text {eff }}$ and $\epsilon$ are numbers uniquely defined for given functions $\varphi_{r, n}(r)$; from the Cauchy inequality, it follows that $\epsilon \geq 0$. 
For 3D systems, the rhs of Eq. (7) should be multiplied by the additional factor $8 / 3$, and the integrals become $I_{\alpha \beta}=\int_{0}^{\infty} d \rho \rho f_{\alpha} f_{\beta}$. Note that for a reciprocal Coulomb interaction, $I_{r r}$ is proportional to the so-called Coulomb logarithm (see, e.g., Refs. [27,28]) and $\Delta_{\text {eff }}=0$; Eq. (7) is then reduced to the classical equation for the temperature relaxation in a plasma [27].

For the constant nonreciprocity [i.e., when $F_{n}(r) / F_{r}(r)=\Delta$ is independent of $\left.r\right]$, we get $\Delta_{\text {eff }}=\Delta$ and $\epsilon=0$. In this case, Eq. (7) has an equilibrium solution given by Eq. (4). Otherwise, we have $\epsilon>0$ and an equilibrium is no longer possible - the temperatures grow with time, approaching the asymptotic solution

$$
t \rightarrow \infty: T_{A}(t)=\tau T_{B}(t)=c t^{2 / 3},
$$

where $c \propto\left(\epsilon n I_{r r}\right)^{2 / 3}$. The asymptotic temperature ratio

$$
\tau=\sqrt{\frac{\left(1+\Delta_{\mathrm{eff}}\right)^{2}+\epsilon}{\left(1-\Delta_{\mathrm{eff}}\right)^{2}+\epsilon}}
$$

is a constant that tends to the equilibrium value

for $\epsilon \rightarrow 0$.

Thus, the disparity $\epsilon$ is the measure of "deviation" from pseudo-Hamiltonian systems, where $\epsilon=0$ and different species reach the detailed dynamic equilibrium with distinct temperatures. For $\epsilon>0$, this remarkable balance is broken and a system acquires energy, so the temperatures continuously grow. Nevertheless, in the next section, we show that even an infinitesimally small damping causes the temperatures to saturate, and then systems with sufficiently small $\epsilon$ behave as "nearly equilibrium."

\section{Effect of damping}

Dynamics of individual particles can be damped due to friction against the surrounding medium. To take friction into account, one has to add the dissipation term $-2 \nu_{A, B}\left(T_{A, B}-T_{b}\right)$ to the rhs of Eq. (7), where $\nu_{\alpha}$ is the respective damping rate in the force $-m_{\alpha} \nu_{\alpha} \mathbf{v}_{\alpha}$ and $T_{b}$ is the background temperature determined by the medium $[21,29]$.

In complex plasmas, the damping is usually very weak: The damping rate is much smaller than the rate of momentum or energy exchange due to interparticle interactions [21]. Let us use Eq. (7) to understand the effect of damping in this regime. For the constant nonreciprocity, we derive the following equilibrium temperatures: $T_{A, B}=$ $T_{b} /(1 \mp \Delta)$ plus small terms proportional to the ratio of $\nu_{A, B}$ to the energy-exchange rate. Thus, to the first approximation, the damping does not affect the equilibrium temperature ratio (4). In a general case, $T_{A, B}$ are no longer growing with time but reach a steady state, since the growth term in Eq. (7) decreases with temperature. The resulting steady-state temperature ratio $\tau_{\nu}$ can be easily derived assuming that $T_{A, B}$ are much larger than $T_{b}$. For similar particles, this condition requires the strong inequality $\nu \ll \epsilon \Delta_{\text {eff }} n I_{r r} / \sqrt{m T_{b}^{3}}$ to be satisfied, which always holds for experiments with 2D complex plasmas discussed below [30]. Then, we obtain the following equation for $\tau_{\nu}$ :

$$
\begin{aligned}
& \tilde{\nu}\left[\left(1-\Delta_{\text {eff }}\right)^{2}+\epsilon\right] \tau_{\nu}^{2}-(\tilde{\nu}-1)\left(1-\Delta_{\text {eff }}^{2}+\epsilon\right) \tau_{\nu} \\
& \quad=\left(1+\Delta_{\text {eff }}\right)^{2}+\epsilon,
\end{aligned}
$$

where $\tilde{\nu}=\nu_{A} / \nu_{B}$. For $\tilde{\nu}=1$, we get $\tau_{\nu}=\tau$, so the steadystate temperature ratio is not affected by friction. Generally, $\tau_{\nu}$ exhibits a weak dependence on $\tilde{\nu}$ : e.g., for the Hertzian interactions (see the next section), the deviation between $\tau_{\nu}$ and $\tau$ is within $\simeq 1 \%$ in the range $0.8 \leq \tilde{\nu} \leq 1.3$.

In colloidal dispersions, where the dynamics is fully damped, the temperatures of both species tend to $T_{b}$. This fact, however, does not imply elimination of nonreciprocity effects: One can easily show that the Brownian dynamics of particles with nonreciprocal interactions (1) is exactly equivalent to the dynamics with conservative interactions (3) and different thermostat temperatures, equal to $T_{b} /(1 \pm \Delta)$ for the $A, B$ species. Therefore, nonreciprocal interactions are expected to have profound effects, e.g., on the dynamic correlations of colloids.

\section{Numerical simulations}

To complement the analytical results and understand the behavior in the strongly coupled $(\Gamma \gg 1)$ regime, we carry out a molecular-dynamics simulation of a 2D binary, equimolar mixture of soft spheres. We implement the velocity Verlet algorithm [31] with an adaptive time step. The simulation box with periodic boundary conditions contains $2 \times 20000$ particles with equal masses. To ensure precise numerical calculations at low and high temperatures, we choose the Hertzian interactions [32,33]. The reciprocal and nonreciprocal parts of the Hertzian potential are given by

$$
\begin{aligned}
\varphi_{r}(r) & =\frac{1}{2}\left(\max \left\{0,1-r / r_{0}\right\}\right)^{2} \varphi_{0}, \\
\varphi_{n}(r) & =\frac{1}{3}\left(\max \left\{0,1-r / r_{0}\right\}\right)^{3} \varphi_{0},
\end{aligned}
$$

where $\varphi_{0}$ is the interaction energy scale and $r_{0}$ is the interaction range. At $t=0$, the particles are arranged into two interpenetrating square lattices with the initial temperature $T_{A}=T_{B}=T_{0}$. (Therefore, at early simulation time, a certain fraction of $T_{0}$ is converted into the interaction energy.)

The numerical results are summarized in Figs. 1 and 2, where the temperature evolution is presented in the dimensionless form (using parameters of the Hertzian potential). 

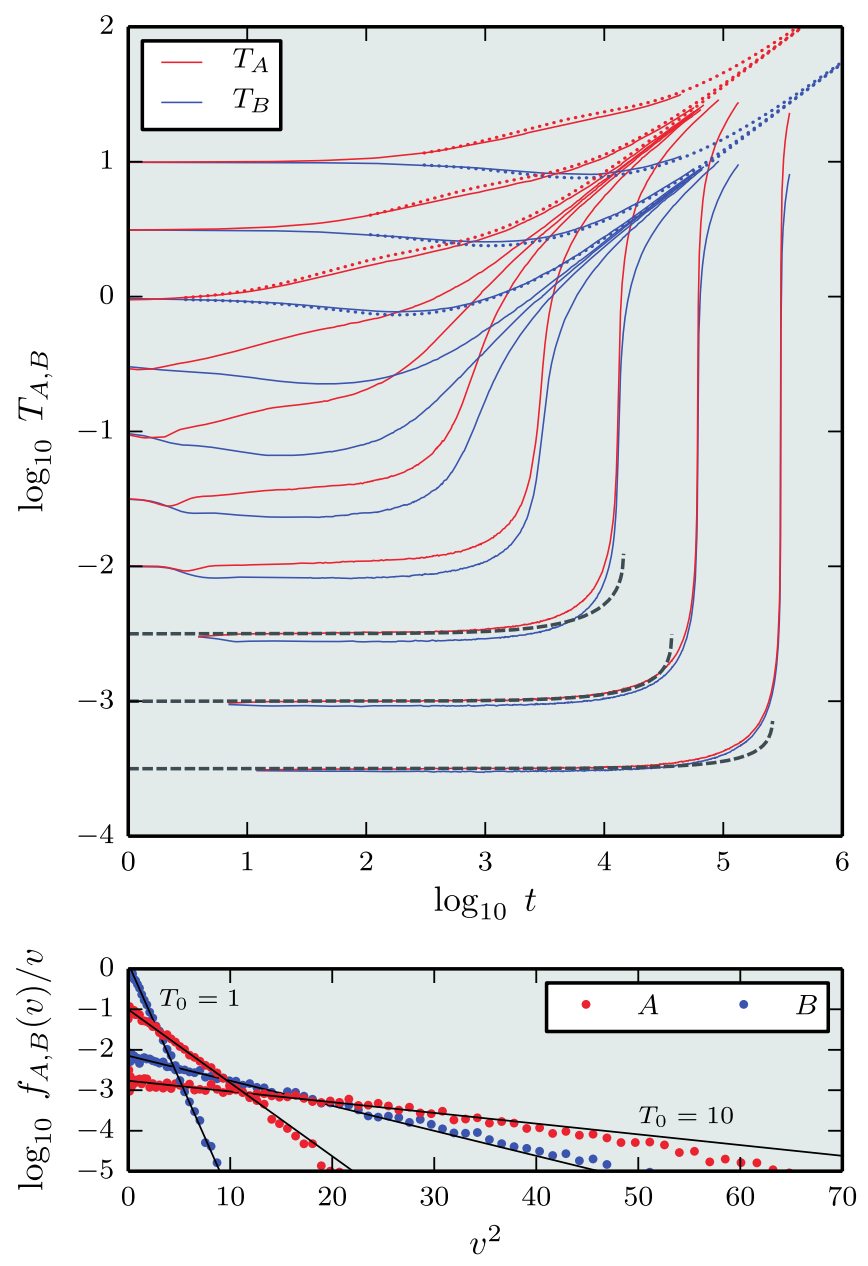

FIG. 1. Growth of the mean kinetic energy in a 2D binary system (no damping). Particles interact via the nonreciprocal Hertzian forces. The upper panel displays the time dependence of the temperatures $T_{A}$ and $T_{B}$. The solid lines show the development obtained from the simulations for the areal fraction $\phi=$ $\pi r_{0}^{2} n=0.3$ and different initial temperatures $T_{0}$. All curves approach the universal asymptotes $\propto t^{2 / 3}$ described by Eqs. (9) and (10). The dotted lines represent the solution of Eq. (7) for $T_{0} \gtrsim 1$. The early development at $T_{0} \ll 1$ is fitted by the explosive solution (13), shown by the dashed lines. The temperatures are normalized by $\varphi_{0}$; time is in units of $\sqrt{m r_{0}^{2} / \varphi_{0}}$. The lower panel illustrates the velocity distributions $f_{A, B}(v)$ at $t \simeq 700$ for $T_{0}=1$ and 10 .

In Fig. 1, we plot the dependencies $T_{A, B}(t)$ for different $T_{0}$. By substituting $F_{r, n}(r)=-d \varphi_{r, n} / d r$ for the Hertzian potential in Eq. (A1) and utilizing Eq. (8), we obtain $\Delta_{\text {eff }}=0.57$ and $\epsilon=0.082$, so Eq. (10) yields the asymptotic temperature ratio $\tau=3.1$. One can see that for all $T_{0}$, the numerical curves approach the expected universal asymptotes described by Eqs. (9) and (10). Note that the early development at sufficiently low temperatures (i.e., when $\Gamma_{0} \propto T_{0}^{-1}$ is sufficiently large) exhibits a sharp dependence on $T_{0}$-we observe the formation of a plateau that broadens dramatically with decreasing $T_{0}$. On the other

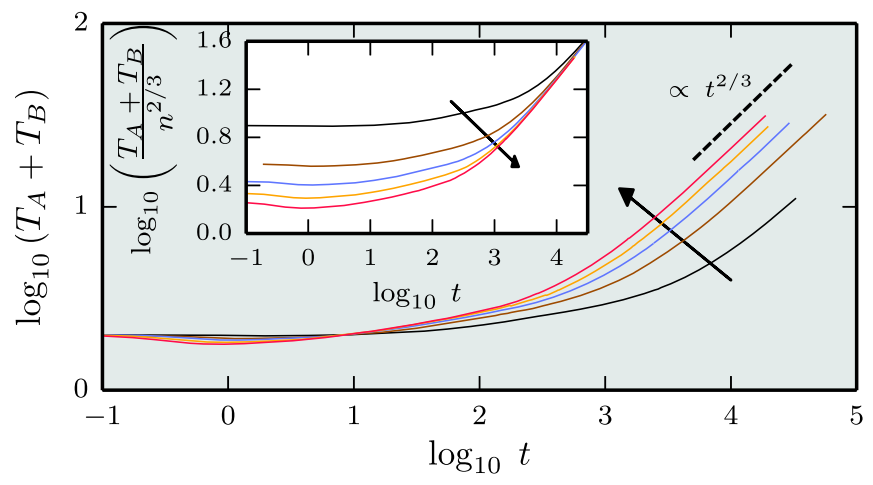

FIG. 2. Time dependence of the total kinetic energy (no damping). The development obtained from the simulations for $T_{0}=1$ and different $\phi(0.1,0.3,0.5,0.7,0.9$, increasing along the arrow) is shown. The inset demonstrates the $\propto n^{2 / 3}$ density scaling of the asymptotic temperature growth. The temperature and time units are the same as in Fig. 1.

hand, for $T_{0} \gtrsim 1$, the numerical results are very well reproduced by the solution of Eq. (7), as expected. A small $(<10 \%)$ deviation observed in this case is due to the fact that weak collisions cannot provide efficient Maxwellization of the velocity distribution for the "hotter" species $A$ (see the lower panel of Fig. 1).

In Fig. 2, we show how the temperature evolution depends on the density $n$. Here, the total kinetic energy $T_{A}(t)+T_{B}(t)$ calculated for different values of the areal fraction $\phi=\pi r_{0}^{2} n$ is plotted. In contrast to the sharp dependence on $T_{0}$ seen in Fig. 1, the increase of $n$ is accompanied by an approximately proportional shortening of the plateau. (A small dip in the early development is due to partial conversion of the initial kinetic energy into the interaction energy.) The inset demonstrates the predicted $\propto n^{2 / 3}$ scaling for the asymptotic temperature growth.

In order to explain the observed behavior at low temperatures, we point out that the approximation of small-angle scattering is not applicable in this strong-coupling regime, and, hence, Eq. (7) is no longer valid. Strong correlations make the analysis rather complicated in this case, but one can implement a simple phenomenological model to understand the essential features. We postulate that at sufficiently low temperatures, the energy growth caused by nonreciprocal interactions can be balanced by nonlinearity, forming a "dynamic potential well" where the system can reside for a long time. Qualitatively, one can then expect the development around the initial temperature to be governed by the activation processes and introduce the effective Arrhenius rate characterizing these processes. Assuming the dimensionless temperature $T$ (normalized by the effective depth of the well) to be small, we employ the following model equation:

$$
\dot{T}=C \exp \left(-T^{-\gamma}\right)
$$


where $C$ is a constant (possible power-law factors can be neglected for $T \ll 1$ ) and $\gamma$ is an exponent determined by the particular form the potential well. Substituting $T^{-\gamma} \simeq$ $T_{0}^{-\gamma}-\gamma T_{0}^{-\gamma-1}\left(T-T_{0}\right)$ in Eq. (12) yields the explosive solution

$$
T(t)=T_{0}-\frac{T_{0}^{\gamma+1}}{\gamma} \ln \left[1-\frac{C \gamma}{T_{0}^{\gamma+1}} \exp \left(-T_{0}^{-\gamma}\right) t\right]
$$

with the explosion time $t_{\mathrm{ex}}=\left(T_{0}^{\gamma+1} / C \gamma\right) \exp \left(T_{0}^{-\gamma}\right)$. In Fig. 1, we demonstrate that at the lowest temperatures, the explosive solution provides quite a reasonable twoparametric fit (with $C=4 \times 10^{-5}$ and $\gamma=0.305$ ) to the numerical results. In the end, we observe a natural crossover of $T_{A, B}(t)$ to the solution of Eq. (7).

When a weak damping is included in the simulations, the temperatures reach a steady state with the ratio given by Eq. (11), as described above. Interestingly, at low temperatures, the system can be dynamically "arrested" due to friction and the asymptotic stage is never reached. A simple analysis of Eq. (12) with the dissipation term shows that the arrest occurs when $\nu t_{\mathrm{ex}} \gtrsim 1$, which is also confirmed by the simulations.

\section{E. Experimental test with complex plasma}

The principal predictions of the theory have been verified in experimental tests performed with weakly damped 2D binary complex plasmas. Such systems are obtained in radio-frequency (rf) discharge chambers [34-37] (see
Appendix B for technical details) sketched in Fig. 3(a). Microparticles injected in a plasma acquire equilibrium negative charges due to absorption of the surrounding electrons and ions [20]. The charging enables particle levitation above a flat horizontal rf electrode, where gravity is balanced by an inhomogeneous electrostatic force-the latter is exerted by a steady vertical electric field ("sheath field") generated in this region. The combination of the two forces provides a stiff vertical confinement for particles, inhibiting their vertical motion. The weak-damping regime is ensured by performing the experiments at low gas pressures.

By injecting two sorts of monodisperse microparticles, it is possible to obtain a quasi-2D mixture. (Unlike earlier experiments with binary complex plasmas [35], we utilize particles with specially chosen combinations of sizes and material densities; see Appendix B.) The particles form two horizontal layers levitated at slightly different heights, so that no vertical pairs are formed.

For the experimental test presented here, the plasma is generated in argon at a pressure of $0.68 \mathrm{~Pa}$. The upper and lower layers are composed, respectively, of melamineformaldehyde spherical particles of diameter $9.19 \pm$ $0.09 \mu \mathrm{m}$ and density $\simeq 1.51 \mathrm{~g} / \mathrm{cm}^{3}$, and polystyrene particles of diameter $11.36 \pm 0.12 \mu \mathrm{m}$ and density $\simeq 1.05 \mathrm{~g} / \mathrm{cm}^{3}$. The mean interparticle distance in each layer is about $\simeq 0.9 \mathrm{~mm}$. The damping rates for the two species, determined by the free-molecular (Epstein) regime of interaction with neutral gas [38], are $\simeq 0.96 \mathrm{~s}^{-1}$ and $\simeq 1.2 \mathrm{~s}^{-1}$, respectively. These
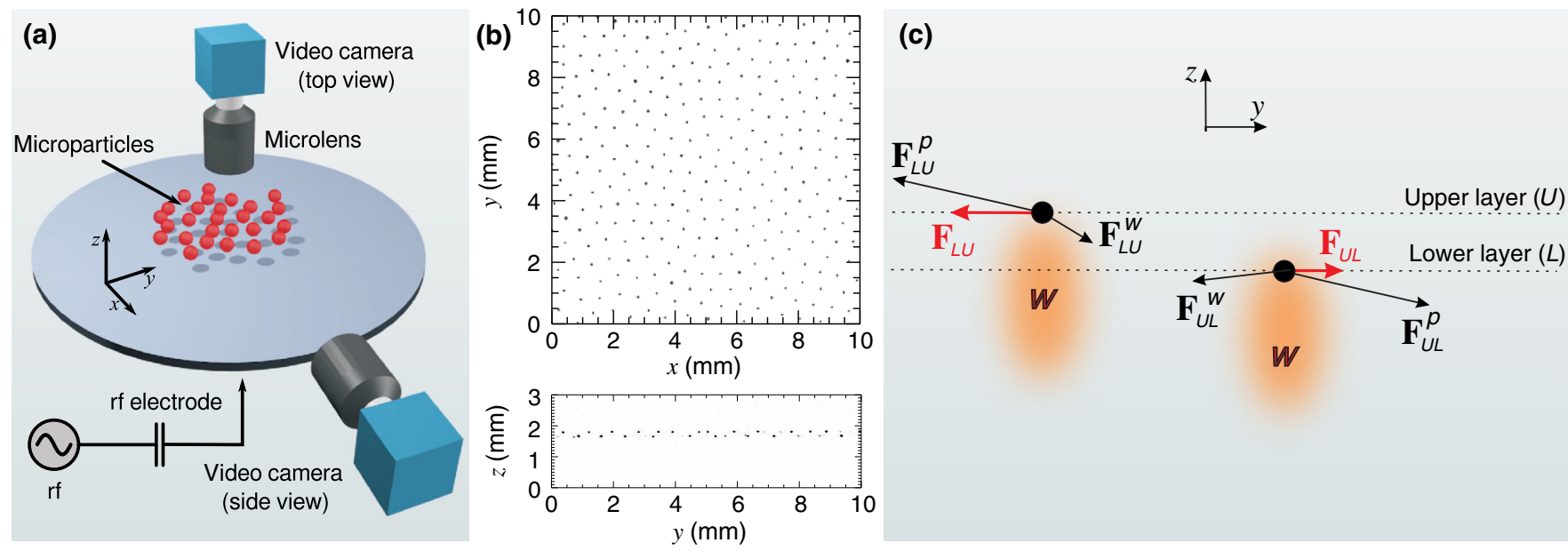

FIG. 3. Scheme of the experimental test and the mechanism of nonreciprocal wake-mediated interactions. (a) Sketch showing the experimental setup. Microparticles levitate above a flat horizontal rf electrode. Two sorts of monodisperse particles form two horizontal monolayers at slightly different levitation heights. The layers are observed with the top-view and side-view video cameras, providing complete information about the position of individual particles. (b) Top and side views of a binary mixture forming the two layers. The respective images are obtained by illuminating the particles with thin horizontal and vertical laser sheets. (c) The total force exerted on the upper-layer $(U)$ particle from the lower-layer $(L)$ particle is the sum of the repulsive force $\mathbf{F}_{L U}^{p}$ of direct interparticle interaction and the attractive force $\mathbf{F}_{L U}^{w}$ from the wake of the lower particle (and similar for the total force on the lower particle). While the direct forces are reciprocal, $\mathbf{F}_{L U}^{p}=-\mathbf{F}_{U L}^{p}$, the wake forces are not, $\mathbf{F}_{L U}^{w} \neq-\mathbf{F}_{U L}^{w}$; since the forces decrease with the distance, we have $\left|\mathbf{F}_{L U}^{w}\right|<\left|\mathbf{F}_{U L}^{w}\right|$ and therefore $\left|\mathbf{F}_{L U}\right|>\left|\mathbf{F}_{U L}\right|$. Thus, the total horizontal forces (relevant for the analysis) can be written as $\mathbf{F}_{L U}=(1+\Delta) \mathbf{F}_{0}$ for the upper layer and $\mathbf{F}_{U L}=-(1-\Delta) \mathbf{F}_{0}$ for the lower layer. 
values are approximately 30-100 times smaller than the characteristic Einstein frequency of microparticles.

Figure 3(b) shows the top-view and side-view images of the layers obtained with the respective video cameras. The height difference $H$ between the layers is varied, from quite small values (when the layers practically merge) to a significant fraction of the horizontal interparticle distance, by tuning the rf power $P_{\text {rf }}$ that effectively controls the sheath field [39].

The sheath field also drives a strong vertical plasma flow, and each microparticle acts as a lens causing the flowing ions to focus downstream from it. This focusing results in the formation of plasma wakes "attached" to particles [19-21,24,40-42]. Figure 3(c) (see also the figure caption) demonstrates how the wakes exert attractive forces and break the action-reaction symmetry for particles levitating in different layers [19,21].

Figure 4 shows that the mean kinetic energy (temperature) of the horizontal motion is noticeably higher for
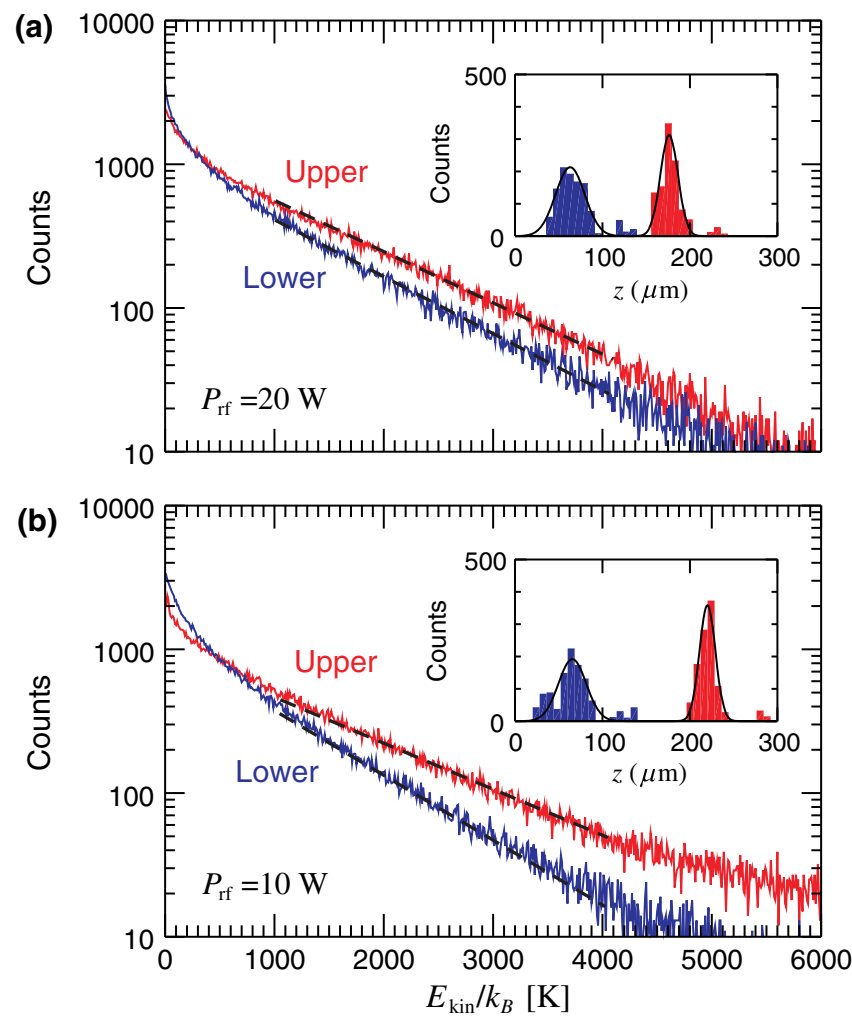

FIG. 4. Kinetic energy distribution for microparticles in monolayers. Shown are the energy distributions measured for two different values of the $\mathrm{rf}$ discharge power $P_{\mathrm{rf}}$, which controls the height difference $H$ between the upper and lower layers. The insets depict the height histograms for particles in the layers; the solid lines are Gaussian fits. (a) $P_{\mathrm{rf}}=20 \mathrm{~W}, H \simeq 110 \mu \mathrm{m}$, and the temperatures of the upper and lower layers are estimated as $T_{U} \simeq 1250 \mathrm{~K}$ and $T_{L} \simeq 1100 \mathrm{~K}$, respectively (from the Maxwellian fit, shown by the dashed lines). (b) $P_{\mathrm{rf}}=10 \mathrm{~W}$, $H \simeq 150 \mu \mathrm{m}$, and the respective temperatures are $T_{U} \simeq 1350 \mathrm{~K}$ and $T_{L} \simeq 1000 \mathrm{~K}$. particles in the upper layer, and the temperature difference between the layers increases with $H$. From Fig. 3(c), we infer that the wake-mediated interactions of particles levitating at different heights are such that nonreciprocity for the upper layer is positive, and for the lower layer it is negative. Since $\Delta$ is an increasing function of $H(\Delta \propto H$ for a small height difference; see Appendix $C$ ), the theory predicts that (i) the upper layer should have a higher temperature than the lower one and (ii) the temperature ratio $T_{U} / T_{L}=(1+\Delta) /(1-\Delta)$ should increase with $H$. We see that both predictions are fully confirmed by the observations.

We point out that while the resolution of the side-view camera used for the experiment is not sufficient to accurately measure the vertical velocities, it shows that the particle motion is almost entirely horizontal. This observation indicates that mechanisms of the individualparticle heating associated with charge fluctuations (which result in the enhancement of the vertical motion; see, e.g., Refs. [43-45]) play a minor role in the experiments.

\section{CONCLUSION}

The presented results provide a basic classification of many-body systems with nonreciprocal interactions. We investigated different nonreciprocity classes in $2 \mathrm{D}$ and $3 \mathrm{D}$ systems that are relevant to a plethora of real situations. For instance, the shadow $[16,18]$ or diffusiophoretic $[10,11]$ interactions have a constant nonreciprocity and can dominate the kinetics of 3D systems, while the forces induced by the flow of the surrounding plasma [21,42] or solvent (depletant) $[12,15]$ are generally characterized by a variable nonreciprocity and govern the action-reaction symmetry breaking in $2 \mathrm{D}$ systems.

Irrespective of the particular nonreciprocity class, all such systems are expected to reveal remarkable behavior. In the weak-damping regime typical-but not limited-to complex plasmas (e.g., nonreciprocal optical forces [8,9] can operate in different systems and do not imply any damping), the reciprocal subensembles reach distinct steady-state temperatures, with the ratio uniquely determined by the effective nonreciprocity. In the opposite fully damped regime typical to colloidal dispersions, the Brownian particle dynamics of the coupled subensembles can be equivalently described with the thermostats having distinct temperatures.

We have verified our theoretical predictions by performing experimental tests with weakly damped 2D binary complex plasmas and expect that similar tests can also be carried out with 3D clouds under microgravity conditions. Furthermore, colloidal dispersions open up a variety of options to probe the effect of nonreciprocal interactions in the strong-damping regime, in particular, by analyzing the dynamic correlations. In this respect, binary suspensions of catalytic colloids are very attractive model systems for 
which the strength of nonreciprocity can be tuned [11]. Another interesting analogy could be found in the purely kinetic clustering transition occurring with one-component microswimmers, which was recently discovered experimentally [46-48] for catalytically driven particles (see also Refs. [49,50]). It is intriguing to check whether the cluster coexisting with a gas of microswimmers acts formally as a second species, at an effective temperature different from that of the surrounding phase. We believe that all these problems constitute promising research topics for the future.

\section{ACKNOWLEDGMENTS}

The authors acknowledge support from the European Research Council under the European Union's Seventh Framework Programme, ERC Grant Agreement No. 267499. M. H. acknowledges support by a fellowship within the Postdoc Program of the German Academic Exchange Service (DAAD). C.-R. D. acknowledges support by the National Natural Science Foundation of China (NNSFC), Grant No. 11405030.

\section{APPENDIX A: PAIR COLLISIONS: VARIATION OF ENERGY AND SCATTERING FUNCTIONS}

We define the relative velocity $\mathbf{v}=\dot{\mathbf{r}}$, the center-of-mass velocity $\mathbf{V}=\dot{\mathbf{R}}$, and their values after a collision $\mathbf{v}^{\prime}=$ $\mathbf{v}+\delta \mathbf{v}$ and $\mathbf{V}^{\prime}=\mathbf{V}+\delta \mathbf{V}$. From Eq. (6), we infer that the relative motion is conservative. Thus, the absolute value of the relative velocity remains unchanged after a collision, $|\mathbf{v}+\delta \mathbf{v}|=|\mathbf{v}|$. Equation (5) governs the variation of the center-of-mass velocity $\delta \mathbf{V}$, which is determined by the relative motion via $\mathbf{F}_{n}(r)$. By employing the relation $\mathbf{v}_{A, B}=\mathbf{V} \pm\left(\mu / m_{A, B}\right) \mathbf{v}$, we obtain the variation of the kinetic energy $E_{A, B}$ after a collision

$$
\begin{aligned}
\delta E_{A, B}= & m_{A, B}\left[\mathbf{V} \cdot \delta \mathbf{V}+\frac{1}{2}(\delta \mathbf{V})^{2}\right] \\
& \pm \mu(\mathbf{V} \cdot \delta \mathbf{v}+\mathbf{v} \cdot \delta \mathbf{V}+\delta \mathbf{V} \cdot \delta \mathbf{v})
\end{aligned}
$$

Since the relative motion is conservative, from Eq. (5), we conclude that $\delta \mathbf{V}$ is parallel to $\delta \mathbf{v}$, i.e., $\delta \mathbf{V} \cdot \delta \mathbf{v}=\delta V \delta v$. For 2D collisions, let us introduce the angle $\theta$ between $\mathbf{V}$ and $\mathbf{v}$, and the scattering angle $\chi$ between $\mathbf{v}^{\prime}$ and $\mathbf{v}$ (Fig. 5). Then, we have $\mathbf{V} \cdot \delta \mathbf{V}=V \delta V \sin \left(\theta-\frac{1}{2} \chi\right), \quad \mathbf{V} \cdot \delta \mathbf{v}=$ $V \delta v \sin \left(\theta-\frac{1}{2} \chi\right)$, and $\mathbf{v} \cdot \delta \mathbf{V}=-v \delta V \sin \frac{1}{2} \chi \quad[26,27]$. For $3 \mathrm{D}$ systems, the corresponding expressions are easily derived using the cosine rule of spherical trigonometry.

In order to calculate the magnitudes of the velocity variations and the scattering angle, we consider the approximation of small-angle scattering $\chi \ll 1$. Using Eqs. (5) and (6), for a given impact parameter $\rho$, we get the following expressions [26]:

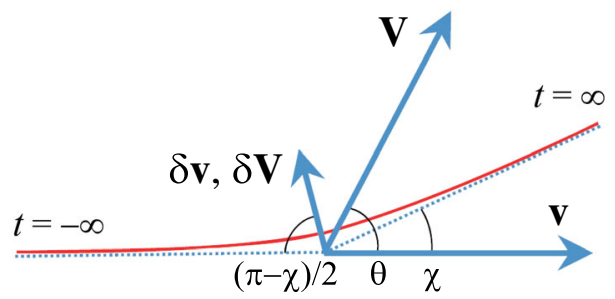

FIG. 5. Sketch illustrating pair collisions in 2D systems. Shown are the variations of the center-of-mass velocity $\mathbf{V}$ and the relative velocity $\mathbf{v}$ as well as the scattering angle $\chi$, plotted in polar coordinates.

$$
\begin{aligned}
\delta V(\rho) & =\frac{4}{M v} f_{n}(\rho), \\
\chi(\rho) & =\frac{\delta v}{v}=\frac{2}{\mu v^{2}}\left[f_{r}(\rho)+\frac{m_{B}-m_{A}}{m_{A}+m_{B}} f_{n}(\rho)\right],
\end{aligned}
$$

determined by the scattering functions $(\alpha=r, n)$

$$
f_{\alpha}(\rho)=\rho \int_{\rho}^{\infty} d r \frac{F_{\alpha}(r)}{\sqrt{r^{2}-\rho^{2}}} .
$$

General equations describing the asymptotic evolution of the mean kinetic temperatures of species $A$ and $B$ are obtained by multiplying $\delta E_{A, B}$ with the collision frequency between the species and averaging it over the Maxwellian velocity distributions [27]. The collision cross section is represented by the integral over the impact parameter [26] $\int d \rho$ for $2 \mathrm{D}$ systems or $\int d \rho 2 \pi \rho$ for $3 \mathrm{D}$ systems. Note that after the integration over $\theta$, all terms in the above expression for $\delta E_{A, B}$ yield contributions $\propto \chi^{2}$.

\section{APPENDIX B: EXPERIMENTAL SETUP}

The experiments are performed in a modified Gaseous Electronics Conference (GEC) rf reference cell, using a low-pressure capacitively coupled plasma discharge [34-37]. The microparticles injected into the plasma acquire negative charges $Q$ and can be levitated in the sheath above the horizontal rf electrode, due to the steady vertical electric field generated in this region. In order to create a "quasimonolayer" binary mixture, we select a particular combination of two sorts of monodisperse microparticles whose material densities $\rho_{A, B}$ and sizes $a_{A, B}$ satisfy the relation $\rho_{A} a_{A}^{2} \simeq \rho_{B} a_{B}^{2}$; the latter is based on the assumption that $Q_{A, B} \propto a_{A, B}$, and the gravity is fully compensated by the electric force (i.e., the contribution of the drag force due to flowing ions [38] is neglected). As the result, the particles of different sorts form two horizontal layers levitated at slightly different heights. By tuning the rf discharge power $P_{\text {rf }}$, it is possible to effectively vary the strength of the vertical confinement [39] and, hence, the height difference $H$. 
A high-resolution video camera Photron FASTCAM 1024 PCI is mounted above the chamber, capturing a top view with a size of $14 \times 14 \mathrm{~mm}^{2}$. In addition, a sideview video camera is used to measure the vertical distance between the layers. The recording rate for the top-view camera is set at 60 frames per second (to assure correct particle velocity measurements, in accordance with recommendations of Refs. [51,52]). The obtained video is analyzed to find the positions of all particles in every frame. The pixel intensity distribution of each particle image is fitted by a 2D Gaussian; its center gives the particle position with subpixel resolution. The particles are traced from frame to frame, and their horizontal velocities are calculated from their positions in two consecutive frames.

\section{APPENDIX C: MODELS FOR WAKE-MEDIATED INTERACTIONS}

All available self-consistent models for the interaction between microparticles in 2D complex plasmas are based on the solution of the kinetic equation for ions moving in the electrostatic field of the sheath, while electrons are described by the Boltzmann distribution [38]. Different approximations used for the ion-collision operator (describing the interaction with neutral gas) merely reflect different experimental regimes (in terms of the rf discharge power and pressure) when the particular model is applicable. Note that the wake generated by a given particle is practically unaffected by the field of the neighbors because the characteristic lateral range of the ion-particle interaction (providing the main contribution to the formation of wake, the Coulomb radius) is typically 1-2 orders of magnitude shorter than the interparticle distance [38].

In order to illustrate the essential features of the wakemediated interaction, we consider a simple point-wake model $[19,53]$. In this model, the wake is treated as a positive, pointlike effective charge $q$ located at the distance $\delta$ below each negatively charged particle (of charge $-Q$ ). So, the total interaction between two particles is a simple superposition of the particle-particle and particle-wake interactions, both described by the (spherically symmetric) Yukawa potentials with effective screening length $\lambda$.

For a binary 2D system of particles, it is convenient to introduce the horizontal (radial) distance $r$ and the vertical distance $z$. The total potential governing the interparticle interactions is $\varphi_{Q}(r, z)-\varphi_{q}(r, z)$, where the particle-particle and particle-wake terms are $\varphi_{Q}(r, z)=\left(Q^{2} / R_{Q}\right) e^{-R_{Q} / \lambda}$ and $\varphi_{q}(r, z)=\left(q Q / R_{q}\right) e^{-R_{q} / \lambda}$, respectively, $R_{Q}=\sqrt{r^{2}+z^{2}}$ is the interparticle distance, and $R_{q}=\sqrt{r^{2}+(z+\delta)^{2}}$ is the distance to the neighboring wake. For the layers separated by the height difference $H$, we readily obtain the potentials of the reciprocal and nonreciprocal forces

$$
\begin{aligned}
\varphi_{r}(r) & =\varphi_{Q}(r, H)-\frac{1}{2}\left[\varphi_{q}(r, H)+\varphi_{q}(r,-H)\right], \\
\varphi_{n}(r) & =\frac{1}{2}\left[\varphi_{q}(r, H)-\varphi_{q}(r,-H)\right] .
\end{aligned}
$$

The interaction is reciprocal for particles levitating in the same layer. For the interlayer interactions, when the height difference is much smaller than the interparticle distance (within each layer), the nonreciprocity parameter scales linearly with $H$, as $\Delta(r) \propto(q / Q) H \delta / r^{2}$.

[1] J. N. Israelachvili, Intermolecular and Surface Forces (Elsevier, Amsterdam, 1992).

[2] M. Dijkstra, R. van Roij, and R. Evans, Effective Interactions, Structure and Isothermal Compressibility of Colloidal Suspensions, J. Chem. Phys. 113, 4799 (2000).

[3] P. G. Bolhuis, A. A. Louis, J. P. Hansen, and E. J. Meijer, Accurate Effective Pair Potentials for Polymer Solutions, J. Chem. Phys. 114, 4296 (2001).

[4] M. Praprotnik, L. Delle Site, and K. Kremer, Multiscale Simulation of Soft Matter: From Scale Bridging to Adaptive Resolution, Annu. Rev. Phys. Chem. 59, 545 (2008).

[5] B. M. Mognetti, P. Virnau, L. Yelash, W. Paul, K. Binder, M. Muller, and L. G. MacDowell, Coarse-Grained Models for Fluids and Their Mixtures: Comparison of Monte Carlo Studies of Their Phase Behavior with Perturbation Theory and Experiment, J. Chem. Phys. 130, 044101 (2009).

[6] K. Hayashi and S. Sasa, The Law of Action and Reaction for the Effective Force in a Non-equilibrium Colloidal System, J. Phys. Condens. Matter 18, 2825 (2006).

[7] P. R. Buenzli and R. Soto, Violation of the Action-Reaction Principle and Self-Forces Induced by Nonequilibrium Fluctuations, Phys. Rev. E 78, 020102(R) (2008).

[8] K. Dholakia and P. Zemanek, Gripped by Light: Optical Binding, Rev. Mod. Phys. 82, 1767 (2010).

[9] E. R. Shanblatt and D. G. Grier, Extended and Knotted Optical Traps in Three Dimensions, Opt. Express 19, 5833 (2011).

[10] B. Sabass and U. Seifert, Efficiency of Surface-Driven Motion: Nanoswimmers Beat Microswimmers, Phys. Rev. Lett. 105, 218103 (2010).

[11] R. Soto and R. Golestanian, Self-Assembly of Catalytically Active Colloidal Molecules: Tailoring Activity Through Surface Chemistry, Phys. Rev. Lett. 112, 068301 (2014).

[12] J. Dzubiella, H. Löwen, and C. N. Likos, Depletion Forces in Nonequilibrium, Phys. Rev. Lett. 91, 248301 (2003).

[13] A. S. Khair and J. F. Brady, On the Motion of Two Particles Translating with Equal Velocities through a Colloidal Dispersion, Proc. R. Soc. A 463, 223 (2007).

[14] C. Mejia-Monasterio and G. Oshanin, Bias- and BathMediated Pairing of Particles Driven through a Quiescent Medium, Soft Matter 7, 993 (2011).

[15] I. Sriram and E. M. Furst, Out-of-Equilibrium Forces between Colloids, Soft Matter 8, 3335 (2012).

[16] V. N. Tsytovich, Dust Plasma Crystals, Drops, and Clouds, Phys. Usp. 40, 53 (1997).

[17] S. A. Khrapak, A. V. Ivlev, and G. E. Morfill, Interaction Potential of Microparticles in a Plasma: Role of 
Collisions with Plasma Particles, Phys. Rev. E 64, 046403 (2001).

[18] M. Chaudhuri, A. V. Ivlev, S. A. Khrapak, H. M. Thomas, and G. E. Morfill, Complex Plasma-The Plasma State of Soft Matter, Soft Matter 7, 1287 (2011).

[19] V. A. Schweigert, I. V. Schweigert, A. Melzer, A. Homann, and A. Piel, Alignment and Instability of Dust Crystals in Plasmas, Phys. Rev. E 54, 4155 (1996).

[20] G. E. Morfill and A. V. Ivlev, Complex Plasmas: An Interdisciplinary Research Field, Rev. Mod. Phys. 81, 1353 (2009).

[21] A. Ivlev, H. Löwen, G. Morfill, and C. P. Royall, Complex Plasmas and Colloidal Dispersions: Particle-Resolved Studies of Classical Liquids and Solids (World Scientific, Singapore, 2012).

[22] D. Helbing and P. Molnar, Social Force Model for Pedestrian Dynamics, Phys. Rev. E 51, 4282 (1995).

[23] D. Helbing, I. Farkas, and T. Vicsek, Simulating Dynamical Features of Escape Panic, Nature (London) 407, 487 (2000).

[24] M. Bonitz, C. Henning, and D. Block, Complex Plasmas: A Laboratory for Strong Correlations, Rep. Prog. Phys. 73, 066501 (2010).

[25] L. Delle Site, Some Fundamental Problems for an EnergyConserving Adaptive-Resolution Molecular Dynamics Scheme, Phys. Rev. E 76, 047701 (2007).

[26] L. D. Landau and E. M. Lifshitz, Mechanics (Pergamon, Oxford, 1976).

[27] E. M. Lifshitz and L. P. Pitaevskii, Physical Kinetics (Pergamon, Oxford, 1981).

[28] L. Spitzer, Physics of Fully Ionized Gases (Dover, New York, 2006).

[29] N. G. van Kampen, Stochastic Processes in Physics and Chemistry (Elsevier, Amsterdam, 1981).

[30] For the Yukawa potential $\varphi_{r}(r)=\left(Q^{2} / r\right) e^{-r / \lambda}$, we get $I_{r r} \sim Q^{4} / \lambda$; so, the rhs of the inequality is proportional to the squared coupling parameter $\Gamma=Q^{2} \sqrt{n} / T_{b}$, which is of the order of $10^{2}-10^{3}$ for typical $2 \mathrm{D}$ experiments [21].

[31] W. C. Swope, H. C. Andersen, P. H. Berens, and K. R. Wilson, A Computer-Simulation Method for the Calculation of Equilibrium-Constants for the Formation of Physical Clusters of Molecules-Application to Small Water Clusters, J. Chem. Phys. 76, 637 (1982).

[32] J. C. Pamies, A. Cacciuto, and D. Frenkel, Phase Diagram of Hertzian Spheres, J. Chem. Phys. 131, 044514 (2009).

[33] L. Berthier, A. J. Moreno, and G. Szamel, Increasing the Density Melts Ultrasoft Colloidal Glasses, Phys. Rev. E 82, 060501(R) (2010).

[34] H. Thomas and G. Morfill, Melting Dynamics of a Plasma Crystal, Nature (London) 379, 806 (1996).

[35] P. Hartmann, Z. Donkó, G. J. Kalman, S. Kyrkos, K. I. Golden, and M. Rosenberg, Collective Dynamics of Complex Plasma Bilayers, Phys. Rev. Lett. 103, 245002 (2009).

[36] V. Nosenko, G. E. Morfill, and P. Rosakis, Direct Experimental Measurement of the Speed-Stress Relation for Dislocations in a Plasma Crystal, Phys. Rev. Lett. 106, 155002 (2011).
[37] C.-R. Du, V. Nosenko, S. Zhdanov, H. M. Thomas, and G. E. Morfill, Interaction of Two-Dimensional Plasma Crystals with Upstream Charged Particles, Europhys. Lett. 99, 55001 (2012).

[38] V. E. Fortov, A. V. Ivlev, S. A. Khrapak, A. G. Khrapak, and G. E. Morfill, Complex (Dusty) Plasmas: Current Status, Open Issues, Perspectives, Phys. Rep. 421, 1 (2005).

[39] V. Steinberg, R. Sütterlin, A. V. Ivlev, and G. Morfill, Vertical Pairing of Identical Particles Suspended in the Plasma Sheath, Phys. Rev. Lett. 86, 4540 (2001).

[40] M. Lampe, G. Joyce, G. Ganguli, and V. Gavrishchaka, Interactions between Dust Grains in a Dusty Plasma, Phys. Plasmas 7, 3851 (2000).

[41] S. V. Vladimirov, S. A. Maiorov, and O. Ishihara, Molecular Dynamics Simulation of Plasma Flow around Two Stationary Dust Grains, Phys. Plasmas 10, 3867 (2003).

[42] R. Kompaneets, U. Konopka, A. V. Ivlev, V. Tsytovich, and G. Morfill, Potential around a Charged Dust Particle in a Collisional Sheath, Phys. Plasmas 14, 052108 (2007).

[43] S. Nunomura, T. Misawa, N. Ohno, and S. Takamura, Instability of Dust Particles in a Coulomb Crystal due to Delayed Charging, Phys. Rev. Lett. 83, 1970 (1999).

[44] O. S. Vaulina, S. A. Khrapak, A. P. Nefedov, and O. F. Petrov, Charge-Fluctuation-Induced Heating of Dust Particles in a Plasma, Phys. Rev. E 60, 5959 (1999).

[45] M. Y. Pustylnik, N. Ohno, S. Takamura, and R. Smirnov, Modification of the Damping Rate of the Oscillations of a Dust Particle Levitating in a Plasma due to the Delayed Charging Effect, Phys. Rev. E 74, 046402 (2006).

[46] I. Theurkauff, C. Cottin-Bizonne, J. Palacci, C. Ybert, and L. Bocquet, Dynamic Clustering in Active Colloidal Suspensions with Chemical Signaling, Phys. Rev. Lett. 108, 268303 (2012).

[47] J. Palacci, S. Sacanna, A. P. Steinberg, D. J. Pine, and P. M. Chaikin, Living Crystals of Light-Activated Colloidal Surfers, Science 339, 936 (2013).

[48] I. Buttinoni, J. Bialké, F. Kümmel, H. Löwen, C. Bechinger, and T. Speck, Dynamical Clustering and Phase Separation in Suspensions of Self-Propelled Colloidal Particles, Phys. Rev. Lett. 110, 238301 (2013).

[49] T. Brotto, J. B. Caussin, E. Lauga, and D. Bartolo, Hydrodynamics of Confined Active Fluids, Phys. Rev. Lett. 110, 038101 (2013).

[50] S. Ebbens, D. A. Gregory, G. Dunderdale, J. R. Howse, Y. Ibrahim, T. B. Liverpool, and R. Golestanian, Electrokinetic Effects in Catalytic Platinum-Insulator Janus Swimmers, Europhys. Lett. 106, 58003 (2014).

[51] Y. Feng, J. Goree, and B. Liu, Errors in Particle Tracking Velocimetry with High-Speed Cameras, Rev. Sci. Instrum. 82, 053707 (2011).

[52] J. D. Williams, E. Thomas, Jr., L. Couëdel, A. V. Ivlev, S. K. Zhdanov, V. Nosenko, H. M. Thomas, and G. E. Morfill, Kinetics of the Melting Front in Two-Dimensional Plasma Crystals: Complementary Analysis with the Particle Image and Particle Tracking Velocimetries, Phys. Rev. E 86, 046401 (2012).

[53] L. Couëdel, V. Nosenko, A. V. Ivlev, S. K. Zhdanov, H. M. Thomas, and G. E. Morfill, Direct Observation of ModeCoupling Instability in Two-Dimensional Plasma Crystals, Phys. Rev. Lett. 104, 195001 (2010). 